\title{
SETD1A Mediated H3K4 Methylation and Its Role in Neurodevelopmental and Neuropsychiatric Disorders
}

\author{
Shan Wang ${ }^{1}$, Anna Bleeck ${ }^{1}$, Nael Nadif Kasri ${ }^{1,2}$, Tjitske Kleefstra ${ }^{2,3}$, Jon-Ruben van \\ Rhijn $^{1 \dagger}$ and Dirk Schubert ${ }^{1 * t}$ \\ ${ }^{1}$ Department of Cognitive Neuroscience, Donders Institute for Brain, Cognition and Behaviour, Radboudumc, Nijmegen, \\ Netherlands, ${ }^{2}$ Department of Human Genetics, Radboudumc, Nijmegen, Netherlands, ${ }^{3}$ Centre of Excellence for \\ Neuropsychiatry, Vincent van Gogh Institute for Psychiatry, Venray, Netherlands
}

OPEN ACCESS

Edited by:

Fabio Coppedè,

University of Pisa, Italy

Reviewed by:

Shigeki Iwase,

University of Michigan, United States Gary Patrick Brennan,

University College Dublin, Ireland

${ }^{*}$ Correspondence:

Dirk Schubert

D.schubert@donders.ru.nl

tThese authors shares last authorship

Specialty section: This article was submitted to

Brain Disease Mechanisms a section of the journal Frontiers in Molecular Neuroscience

Received: 08 September 2021 Accepted: 12 October 2021 Published: 03 November 2021

Citation:

Wang S, Bleeck A, Nadif Kasri N, Kleefstra T, van Rhijn J-R and Schubert D (2021) SETD1A Mediated H3K4 Methylation and Its Role in

Neurodevelopmental and

Neuropsychiatric Disorders.

Front. Mol. Neurosci. 14:772000. doi: 10.3389/fnmol.2021.772000
Posttranslational modification of histones and related gene regulation are shown to be affected in an increasing number of neurological disorders. SETD1A is a chromatin remodeler that influences gene expression through the modulation of mono- di- and trimethylation marks on Histone-H3-Lysine-4 (H3K4me1/2/3). H3K4 methylation is predominantly described to result in transcriptional activation, with its mono- di- and trimethylated forms differentially enriched at promoters or enhancers. Recently, dominant mostly de novo variants in SETD1A have clinically been linked to developmental delay, intellectual disability (DD/ID), and schizophrenia (SCZ). Affected individuals often display both developmental and neuropsychiatric abnormalities. The primary diagnoses are mainly dependent on the age at which the individual is assessed. Investigations in mouse models of SETD1A dysfunction have been able to recapitulate key behavioral features associated with ID and SCZ. Furthermore, functional investigations suggest disrupted synaptic and neuronal network function in these mouse models. In this review, we provide an overview of pre-clinical studies on the role of SETD1A in neuronal development. A better understanding of the pathobiology underlying these disorders may provide novel opportunities for therapeutic intervention. As such, we will discuss possible strategies to move forward in elucidating the genotype-phenotype correlation in SETD1A associated disorders.

Keywords: SETD1A, neurodevelopmental disorders (NDD), psychiatric disorders, chromatin modification, histone methlyation, schizophrenia

\section{INTRODUCTION}

Chromatin modification and the related regulation of gene expression patterns have been linked to several neurological disorders, in particular neurodevelopmental (NDD) or neuropsychiatric disorders (NPD; Gabriele et al., 2018; Satterstrom et al., 2020; Mossink et al., 2021). The basic building block for chromatin is the nucleosome, which consists of a 147 base pair DNA structure that wraps around an octamer of the four core histones $\mathrm{H} 3, \mathrm{H} 4, \mathrm{H} 2 \mathrm{~A}$, and $\mathrm{H} 2 \mathrm{~B}$. Chromatin structure dynamics are closely associated with DNA accessibility and the efficiency of DNA 
transcription and replication. Currently, it is well-recognized that epigenetic mechanisms such as post-translational modification of histones can control chromatin structure and organization, thereby influencing gene expression (Mossink et al., 2021).

One such posttranslational chromatin modification is the methylation of lysine groups at histones through different enzymes. Here we focus on mono-, di- and trimethylation of lysine 4 at histone $\mathrm{H} 3(\mathrm{H} 3 \mathrm{~K} 4 \mathrm{me} 1 / 2 / 3)$. $\mathrm{H} 3 \mathrm{~K} 4$ methylation is generally implicated in transcription (Kusch, 2012). H3K4me1, 2, and 3 localizes to specific parts of the nucleosome: $\mathrm{H} 3 \mathrm{~K} 4 \mathrm{me} 1$ is distributed at enhancer regions, $\mathrm{H} 3 \mathrm{~K} 4 \mathrm{me} 2$ is found in nucleosomes further downstream in the body of genes, and $\mathrm{H} 3 \mathrm{~K} 4 \mathrm{me} 3$ is located in nucleosomes near the transcription start sites (TSS) of expressed genes, presumably at promoter regions (Kusch, 2012). Over the past decades, enzymes of the type 2 lysine methyltransferase (KMT2, also known as mixed lineage leukemia; MLL) family have been found responsible for bulk $\mathrm{H} 3 \mathrm{~K} 4$ methylation. This is a highly conserved family, composed of six members. These six genes all contain a Su(var)3-9, Enhancer-of-zeste and Trithorax (SET) and post-SET domain, which are together responsible for the proteins' methyltransferase activity and enable regulation of important aspects of cell physiology and development (Crump and Milne, 2019).

In this review, we will focus on SETD1A (also known as KMT2F), the main mammalian H3K4me1/2/3 methyltransferase. Current research associates SETD1A dysfunction with neurodevelopmental disorders (NDDs), early onset epilepsy, and schizophrenia (SCZ; Singh et al., 2016; Yu et al., 2019; Kummeling et al., 2020). This suggests that SETD1A plays a crucial role both during brain development as well as in maintaining healthy brain function.

\section{THE FUNCTION OF SETD1A IS HIGHLY CONSERVED THROUGHOUT EVOLUTION}

The methyltransferase activity of SETD1A is dependent on its interactions with several other proteins, which form a highly conserved complex, designated the "complex of proteins associated with Set1" (COMPASS). Originally identified in yeast (Ruthenburg et al., 2007), COMPASS complexes remain rather conserved during evolution: in Drosophila there are three Set1-like H3K4 methyltransferase complexes with three different enzymatic subunits: Set1, Trithorax (Trx), and Trithorax-related (Trr), whereas mammals have six Set1-like H3K4 methyltransferases: SETD1A/B and MLL1-4. Based on the sequence homology of the SET-containing enzymatic subunits and composition of the COMPASS, it was defined that MLL1/2 are homologous to Trx, MLL3/4 are homologous to Trr, and SETD1A/B are homologous to dSet1 (Mohan et al., 2011). The conserved structure of COMPASS complexes is also reflected in the function of the proteins, with Set1 in Drosophila and SETD1A/B in mammals both being considered as the major H3K4 trimethyl transferases (Ardehali et al., 2011; Clouaire et al., 2012). This underscores the essential biological function of SETD1A.

\section{SETD1A REGULATES GENE TRANSCRIPTION AS PART OF A MULTI-SUBUNIT PROTEIN COMPLEX}

In humans, SETD1A contains highly conserved SET and post-SET domains at the $\mathrm{C}$ terminus, like all other members of the KMT2 family (Figure 1A). Additionally, adjacent to the SET domain in the N-terminal direction, the n-SET domain (including the conserved WDR5 binding "WIN" motif) plays an important role in $\mathrm{H} 2 \mathrm{~B}$ ubiquitylation and eventually downstream H3K4 methylation (Kim et al., 2013). Near the N-terminal region, SETD1A also contains an RNA recognition motif (RRM) domain. All mammalian SET1-family complexes constitute WDR5, RBBP5, ASH2, and DPY30 forming the four subunit sub-complex WRAD, which is essential for H3K4 methyltransferase activity (Ernst and Vakoc, 2012; Figure 1B). Whereas WRAD is an essential sub-complex for members of the SET1-family in general, the functional SETD1A complex requires additional subunits. Such additional proteinprotein complexes are formed with CFP1, WDR82, and HCF1 (Figure 1B). CFP1, also known as CXXC1, serves as the predominant targeting module for the SETD1A complex and plays a key role in guiding $\mathrm{H} 3 \mathrm{~K} 4 \mathrm{me} 3$ deposition and proper expression of target genes (Brown et al., 2017). WDR82 interacts with SETD1A via the RRM domain. It mediates binding to the Ser5-phosphorylated C-terminal domain of RNA polymerase II, which results in the initiation of transcription by recruiting the SETD1A complex to transcription start sites (Lee and Skalnik, 2008). Lastly, HCF1 interacts with SETD1A through the HCF-1-binding motif (HBM). Through this interaction, the SETD1A complex is recruited to E2F-responsive promoters, where it can induce histone methylation and transcriptional activation and is involved in the regulation of cell cycle-related mechanisms (Tyagi et al., 2007). Taken together, this suggests that SETD1A can perform a multitude of biological functions, depending on specific interactions between subunits within the complex.

\section{SETD1A DYSFUNCTION IN NEURODEVELOPMENTAL AND NEUROPSYCHIATRIC DISORDERS}

All six human KMT2 family complexes are widely expressed in different tissues and exhibit non-redundant cellular functions (Vallianatos and Iwase, 2015). This may explain why mutations in each of the different KMT2 family members have been shown to be causally linked to different syndromes and disorders (Vallianatos and Iwase, 2015). For example, mutations in KMT2A $(M L L 1)$ are associated with Wiedemann-Steiner syndrome, KMT2C (MLL3) with Kleefstra syndrome spectrum (Kleefstra et al., 2012; Frega et al., 2020), whereas KMT2D (MLL4, in humans, also called MLL2) mutations lead to Kabuki Syndrome 1 (Min Ko et al., 2017; Sobreira et al., 2017; Sun et al., 2017). In several studies with the focus on de novo mutations in humans, loss of function (LoF) of SETD1A was identified as a high-risk contribution to 


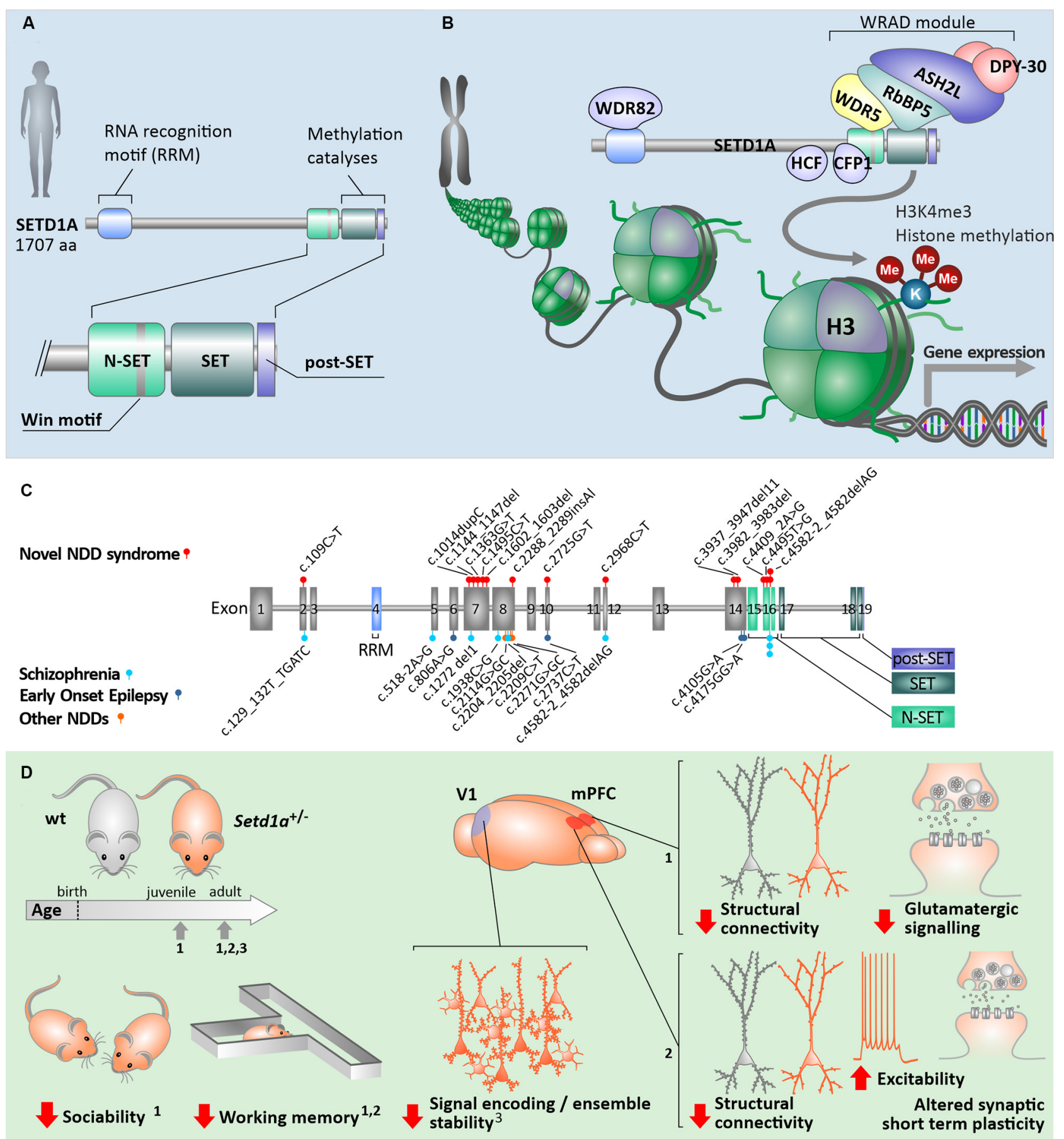

FIGURE 1 | Human SETD1A in health and neurological disorders. (A) General build-up of the human SETD1A protein and its key binding/catalytic motifs. (B) Principle of H3K4me3 histone modification via the COMPASS protein complex with SETD1A. (C) Known mutations in the coding sequence of SETD1A and associated neurological disorders [data from Singh et al. (2016); Yu et al. (2019); Kummeling et al. (2020)]. (D) Consequences of Setd1a haploinsufficiency in $\operatorname{Setd}_{1} \mathrm{a}^{+/}$adult mouse models on behavior as well as neuronal (network) structure and function, compared to wildtype animals (wt). The relevant studies were performed at different developmental timepoints, each of which indicated by arrows on the developmental timeline. Numbers indicate the referenced sources: data from Nagahama et al. (2020) (1); (Mukai et al., 2019) (2); (Hamm et al., 2020) (3); V1 - primary visual cortex, mPFC-medial prefrontal cortex.

the etiology of SCZ (Takata et al., 2014; Singh et al., 2016; Birnbaum and Weinberger, 2017). Initially, this led to the proposition that reduced SETD1A function is a monogenic cause of SCZ. More recently this conceptual linking has been extended by associating mutations in SETD1A with disorders that are clinically characterized as NDDs, symptomatically 
represented with developmental delay, intellectual disability, behavioral problems as well as early onset epilepsy (Yu et al., 2019; Kummeling et al., 2020). Thus different individuals with SETD1A deficiency can show hallmarks of either impaired brain development and/or SCZ (Kummeling et al., 2020).

So far, the majority of individuals with a SETD1A mutation that report developmental problems were recruited at pediatric ages, whilst individuals reported with variants associated with SCZ were recruited at adolescent/adult ages. Specific symptoms for individuals diagnosed with SCZ typically show a later onset during the lifetime and are composed of a variation of delusions and hallucinations in addition to cognitive impairment and a decrease in social skills (Owen et al., 2016). Moreover, SETD1A-deficient individuals diagnosed with developmental problems have increasing symptoms typically associated with neuropsychiatry, including aggressive behaviors and anxiety, whereas some individuals diagnosed with SCZ also exhibited learning difficulties, which are more typically regarded as developmental problems (Owen et al., 2016; Singh et al., 2016). Therefore it is likely that SETD1A deficiency is associated with a variable longitudinal course and phenotypic spectrum across the lifespan. Taken together, LoF of SETD1A may lead to a neurodevelopmental syndrome that includes neuropsychiatric phenotypes typical for SCZ (Singh et al., 2016; Kummeling et al., 2020) and some individuals diagnosed with an NDD at pediatric ages might develop SCZ later in life.

Heterozygous mutations in the SETD1A gene, which is located on chromosome 16p11.2, have been shown to occur in multiple exons along the gene, predominantly 5 ' but not within the catalytic SET domain. These mutations are suggested to result in LoF of the domain responsible for the methylation activity of the protein (Figure 1C; Table 1). Other mutations, located more upstream in the gene, are mostly frameshift mutations predicted to lead to a premature stop codon and reduced SETD1A expression, without a dominant negative function induced by the mutant allele (Cameron et al., 2019). Most of the known mutations are de novo (Table 1), and there are two variants (c.2968C $>\mathrm{T}$; c.4582-2delAG) that have been reported in both developmental disorders and SCZ (Singh et al., 2016; Kummeling et al., 2020). Currently, there is no convincing evidence for the specific type of mutation nor the exon loci of the various SETD1A mutations to be reliable predictors for the clinical consequences of the affected individual (Singh et al., 2016; Yu et al., 2019; Kummeling et al., 2020). Insight into the specific molecular and cellular (neuronal) mechanisms affected by LoF of SETD1A will shed more light on how neurological disorders might be established in the brain.

\section{ROLE OF SETD1A IN NEURODEVELOPMENT-LESSONS FROM RODENT MODELS}

Studying mouse models for SETD1A deficiency has recently provided important information about the etiology of SETD1A associated neurological disorders. Recent studies focused on two different genetic mouse models for Setd1a haploinsufficiency. While one model mimics a human de novo frameshift mutation in exon 7 (Nagahama et al., 2020), a second model contains a LacZ/Neo cassette upstream of exon 4 that leads to LoF of Setdla (Mukai et al., 2019; Hamm et al., 2020). Whereas homozygous mutations in Setd1a are lethal (Bledau et al., 2014), Setd1 $a^{+/}$mice of both models are viable and display distinct behavioral phenotypes (Figure 1D). Setd1 $a^{+/-}$mice express impairments in social behavior as well as in working memory and learning (Mukai et al., 2019; Nagahama et al., 2020) which resemble some of the known deficits in human individuals with SETD1A haploinsufficiency, i.e., reductions in social skills, learning difficulties and cognitive impairments (Owen et al., 2016; Singh et al., 2016; Kummeling et al., 2020). Interestingly, Setd $1 a^{+/-}$mice with an LoF in exon 7 also show hyperactivity, deficiency in novel object recognition, and impaired avoidance from aversive situations (Nagahama et al., 2020). Such behavioral phenotypes have not been reported for Setd $1 a^{+/-}$mice with an LoF in exon 4 (Mukai et al., 2019; Hamm et al., 2020). It remains unresolved whether these differences in behavior are related to the exon loci of the mutation, the animal strains, or to differences in the study design.

Overall, even though differences between mouse models may exist, in rodents Setd1a haploinsufficiency seems to result in alterations of working memory and learning, as well as deficits in sociality. These consequences of SETD1A deficiency seem to span across evolution, as deficits in associative learning have been reported in Drosophila with a loss of the SETD1A homolog Set1 as well (Kummeling et al., 2020).

\section{NEURONAL PHENOTYPES OF SETD1A DYSFUNCTION}

Although it is evident that SETD1A mutations cause biological vulnerability to a broad neurodevelopmental phenotypic spectrum, the underlying cellular and molecular mechanisms remain poorly understood. Nevertheless, Setd $1 a^{+/-}$rodent models have already revealed information about the function of SETD1A during neuronal development and gave us the first insight into how Setdla mutations may result in observed behavioral phenotypes.

SETD1A cooperates with Histone Cell Cycle Regulator (HIRA), an epigenetic regulator involved in neurogenesis (Li and Jiao, 2017). Together, these two regulators increase $\mathrm{H} 3 \mathrm{~K} 4 \mathrm{me}$, leading to an increase in $\beta$-catenin, which is a key component of the canonical $\mathrm{Wnt} / \beta$-catenin pathway. $\mathrm{Wnt} / \beta$ catenin signaling plays an important role in promoting neural stem cell proliferation whereas inhibiting differentiation into mature neurons (Zhang et al., 2011). Concordantly, deletion of Setd1a severely affects the proliferation of not only embryonic stem cells (ESCs) but also epiblast stem cells, neuronal stem cells, and induced pluripotent stem cells (iPSCs), possibly by influencing the cell cycle distribution through an increased G1 phase time but a reduced S phase (Bledau et al., 2014). Additionally, ESCs with Setd1a deletion fail to differentiate entirely, suggesting severe differentiation deficits (Bledau et al., 
TABLE 1 | SETD1A species-specific properties and mutation locations in the Human SETD1A gene.

\section{Species-specific properties of SETD1A}

\begin{tabular}{|c|c|c|c|c|}
\hline & Human & Mouse & Drosophila & Yeast \\
\hline Gene & SETD1A, KMT2F, KIAA0339 & Setd1a, KMT2F, mKIAA0339 & Set1 & SET1, KMT2 \\
\hline Chromosome & $16 \mathrm{p} 11.2$ & $7 F 3$ & $3 \mathrm{~L}$ & VIII \\
\hline Ensemble ID & ENSG00000099381 & ENSMUSG00000042308 & FBgn0040022 & YHR119W \\
\hline Transcript length (bps) & 5,991 & 6,487 & 5,495 & 3,243 \\
\hline Exons & 19 & 19 & 6 & 1 \\
\hline Protein length (amino acids) & 1,707 & 1,716 & 1,641 & 1,080 \\
\hline Protein size (kDa) & 186 & 186 & 188 & 123 \\
\hline
\end{tabular}

\begin{tabular}{|c|c|c|c|}
\hline \multicolumn{4}{|c|}{ Mutation locations described for human SETD1A } \\
\hline Exon location & Mutation & Type & Associated phenotype \\
\hline 2 & c.129_132T>TGATC & frameshift & $\mathrm{SCZ}^{1}$ \\
\hline 2 & c. $109 \mathrm{C}>\mathrm{T}$ & nonsense & $\mathrm{NDD}^{2}$ \\
\hline 5 & c. $518-2 A>G$ & splice acceptor & $\mathrm{SCZ}^{1}$ \\
\hline 6 & c. $806 A>G$ & missense & Epilepsy $^{3}$ \\
\hline 7 & c.1272del1 & frameshift & $\mathrm{SCZ}^{1}$ \\
\hline 7 & c.1014dupC & frameshift & $\mathrm{NDD}^{2}$ \\
\hline 7 & c.1144_1147del & frameshift & $\mathrm{NDD}^{2}$ \\
\hline 7 & c. $1363 \bar{G}>T$ & nonsense & $\mathrm{NDD}^{2}$ \\
\hline 7 & c. $1495 \mathrm{C}>\mathrm{T}$ & nonsense & $\mathrm{NDD}^{2}$ \\
\hline 7 & c.1602_1603del & frameshift & $\mathrm{NDD}^{2}$ \\
\hline 8 & C. $1938 C>G$ & stop gained & $\mathrm{SCZ}^{1}$ \\
\hline 8 & c. $2114 G>G C$ & frameshift & $\mathrm{NDD}^{1}$ \\
\hline 8 & c.2204_2205del & frameshift & $\mathrm{SCZ}^{1}$ \\
\hline 8 & c.2288_2289insA & frameshift & $\mathrm{NDD}^{2}$ \\
\hline 8 & c. $2209 \mathrm{C}>\mathrm{T}$ & stop gained & $\mathrm{NDD}^{1}$ \\
\hline 8 & c. $2271 G>G C$ & frameshift & $\mathrm{NDD}^{1}$ \\
\hline 10 & c. $2725 \mathrm{G}>\mathrm{T}$ & nonsense & $\mathrm{NDD}^{2}$ \\
\hline 10 & c. $2737 \mathrm{C}>\mathrm{T}$ & missense & Epilepsy $^{3}$ \\
\hline 12 & c. $2968 C>T$ & stop gained & $\mathrm{SCZ}^{1}, \mathrm{NDD}^{1,2}$ \\
\hline 14 & c.3937_3947del11 & frameshift & $\mathrm{NDD}^{2}$ \\
\hline 14 & c.3982_3983del & frameshift & $\mathrm{NDD}^{2}$ \\
\hline 14 & c. $4105 \mathrm{G}>\mathrm{A}$ & missense & Epilepsy $^{3}$ \\
\hline 14 & c. $4175 \mathrm{G}>\mathrm{A}$ & missense & Epilepsy $^{3}$ \\
\hline 15 & c. $4409-2 A>G$ & splice acceptor & $\mathrm{NDD}^{2}$ \\
\hline 15 & c. $4495 \mathrm{~T}>\mathrm{G}$ & missense & $\mathrm{NDD}^{2}$ \\
\hline 16 & c.4582-2delAG>- & splice acceptor & $\mathrm{SCZ}^{1}, \mathrm{NDD}^{1,2}$ \\
\hline
\end{tabular}

1.(Singh et al., 2016); 2. (Kummeling et al., 2020); 3. (Yu et al., 2019).

2014). This indicates that SETD1A deficiency is associated with a disturbed balance between proliferation and differentiation, possibly through $\mathrm{Wnt} / \beta$-catenin signaling. This balance is important for early cortical development and defects herein are thought to be an underlying cause for developmental disorders (Ernst, 2016). Taken together, altered neuronal proliferation and differentiation could contribute to the NDD phenotypes found in individuals with SETD1A haploinsufficiency. The understanding of how and to which extent SETD1A may also influence neuronal migration remains limited. However, recently it was shown that by introducing a missense mutation p.R913C on exon 10 of SETD1A into E14.5 embryonic mouse brain, neurons migrated faster to the superficial cortical layers when assessed at birth (Yu et al., 2019). This suggests that this specific mutation may disturb the normal process of cortical development and may lead to long-term consequences for the formation of neuronal circuits. Further studies focusing on other mutation types such as frameshift mutations or other mutation loci are needed to better understand the role of SETD1A in neuronal migration.
Deficits in neurogenesis and potentially altered neuronal migration associated with SETD1A deficiency raise questions about the consequences for neuronal network organization and communication. In the prefrontal cortex of mice, Setdla mRNA can be detected at various developmental stages, from E14.5 until 4 months postnatally (Mukai et al., 2019; Yu et al., 2019). SETD1A protein predominantly expresses in neurons, not glial cells, and such SETD1A-positive neurons are distributed over all cortical layers except L1 (Mukai et al., 2019). Synaptogenesis is a key developmental process for neuronal circuitry formation in both humans and rodents, with the critical period of synaptogenesis occurring during the first 3 postnatal weeks in rodents (Semple et al., 2013). Therefore disruption in SETD1A function in particular within this time window is supposed to result in abnormal neuronal network organization. Indeed, Setd $1 a^{+/-}$mice show reduced neuronal connectivity by means of reduced spine density, particularly mushroom spine density on pyramidal neurons (Mukai et al., 2019; Nagahama et al., 2020; Figure 1D). Dysregulated spine density is known to be associated with multiple neurological 
disorders, such as Down syndrome and SCZ (Geschwind and Levitt, 2007; Nishiyama, 2019; Lo and Lai, 2020). Furthermore, reduced axonal projections patterns of cortical neurons from Setd $1 a^{+/-}$mice have been suggested to result in changes in axonal connectivity (Mukai et al., 2019). Taken together, these data implicate that Setdla deficiency during rodent brain development results in deficits in general neuronal circuitry formation.

The structural neuronal circuitry abnormalities observed in Setd1a $a^{+-}$mouse models have also been associated with altered neuronal communication. On the one hand, Setd1a haploinsufficiency could be related to enhanced intrinsic neuronal excitability (Mukai et al., 2019), on the other hand, there is accumulating evidence for disturbed synaptic function in adolescent as well as adult Setd1 $a^{+/-}$mice (Figure 1D). Whereas cortical L2/3 pyramidal neurons of Setd1 $a^{+/-}$mice have been found to receive normal inhibitory inputs from spontaneously active networks, in such neurons the excitatory drive appears to be significantly reduced (Nagahama et al., 2020). This reduction seems to be elicited by changes in both the number of functional excitatory synapses present as well as the strength of the excitatory inputs. It is to be expected that altered neuronal excitability and impaired excitatory glutamatergic communication result in altered neuronal signal processing. Indeed, aberrant ensemble activity and oscillations in the primary visual cortex of Setd1a $a^{+/}$mice were previously identified, which could contribute to disruptions in sensory processing circuits (Hamm et al., 2020). This finding may offer circuitlevel evidence for sensory-processing dysfunctions observed in neuropsychiatric disorders.

Furthermore, in mature cortical circuitries of Setd $1 a^{+/-}$mice, changes in excitatory synaptic short-term plasticity have been observed, such as an increase in short-term depression (Mukai et al., 2019). There is currently no evidence for altered long-term plasticity in Setd $1 a^{+/-}$mice. However, functional data indicates Setd $1 a^{+/-}$mice show alterations in their NMDAR subunit composition, which are key contributors to NMDAR-dependent long-term potentiation of synaptic signaling (Nagahama et al., 2020). Since synaptic plasticity, including short-term plasticity, is regarded as the cellular basis for learning and memory (Elgersma and Silva, 1999; Jaaskelainen et al., 2011), altered signal processing in combination with altered synaptic plasticity as observed in mature neuronal networks of Setd $1 a^{+/-}$mice may be a contributing factor to the learning deficits observed from patients with SETD1A mutation.

Several molecular mechanisms may lead to structural and functional neuronal circuitry alterations in $\operatorname{Setd} 1 a^{+/-}$mice. SETD1A, as an epigenetic regulator, is involved in the regulation of downstream gene expression. In cross-species investigations, it has been shown that Setdla deficiency leads to significant changes in the transcriptomic profile of rodents (Mukai et al., 2019; Yu et al., 2019; Nagahama et al., 2020), and humans (Cameron et al., 2019). Both upregulation and downregulation of SETD1A target genes have been observed. Targets of SETD1A are highly expressed in pyramidal neurons, and dysregulated genes caused by Setd1a deficiency are enriched in annotations associated with synaptic functions such as "synapse organization" and "chemical synaptic transmission" (Mukai et al., 2019). Several genes important for the formation of excitatory synapses, such as Homer1, PTPRO, and ABI1, are downregulated in the mPFC of Setdla ${ }^{+/}$mice (Nagahama et al., 2020), whereas SLITRK4, which is important for neurite outgrowth and excitatory synapse formation, has been found to be upregulated (Mukai et al., 2019). This upregulation of SLITRK4 may contribute to the observed alterations in neuronal morphology and spine densities in Setd $1 a^{+/-}$mice.

Thus, current research suggests a crucial role of SETD1A in the development and maintenance of neuronal network function. These insights from rodent models with SETD1A haploinsufficiency may shed light on the question of how SETD1A deficiency may result in neurophysiological and clinical phenotypes.

\section{AN OUTLOOK TOWARDS HUMAN MODELS FOR NEUROPSYCHIATRIC DISORDERS}

Current data provide strong evidence for reduced SETD1A expression being causative for neurodevelopmental and neuropsychiatric disorders in humans. However, the biological mechanisms affected by SETD1A and histone methylation have only recently been unraveled and current knowledge has been limited to animal models. These models can provide essential and valuable insight into general mechanisms that may underlie disease and they also allow testing behavioral interventions. However, they still lack translational power. This is especially relevant in the context of epigenetic regulation as well as neuropsychiatric disorders such as SCZ, which often show highly human-specific phenotypes. Therefore, research in a human neuronal context could eventually provide deeper insight into the molecular processes affected by SETD1A deficiency.

Human iPSCs are a promising tool that revolutionized the way of human disease modeling. iPSCs have been applied to the study of a large number of diseases and pioneered the concept of "disease in a dish" (Shi et al., 2017). Human neuronal networks derived from patient iPSCs or from iPSCs with introduced disease-related mutations allow investigations in human genetic background. Currently, there are no published data on patients or genetically modified iPSC models with SETD1A deficiency. However, functional and molecular phenotyping of SETD1A deficient human iPSCsderived neuronal networks could further elucidate how SETD1A affects gene expression associated with particular aspects of neuronal function and network maturation in human neuronal circuitry. Furthermore, such a platform can be used to investigate novel agents (Sohal and Rubenstein, 2019) that can enhance the function of SETD1A, which are of interest for the development of novel therapeutic interventions. One of the main challenges in the field of drug development is that we lack a complete understanding of the way epigenetic modification of histone marks can modulate neuronal function (Berger, 2007). For example, there is crosstalk between H3K9me 
and H3K4me (Li et al., 2008; Matsumura et al., 2015), which indicates that chemicals regulating $\mathrm{H} 3 \mathrm{~K} 9$ me can possibly also influence H3K4me. Drug interventions in Setd1 $1 a^{+/}$rodent models showed that treatment with the demethylation inhibitor ORY1001 in adult mice could rescue cognitive and circuitry deficits (Mukai et al., 2019). This indicates that pharmacological interventions may hold therapeutic potential also on the established and matured brain. Further investigation is however required to better characterize the potential of ORY1001 for clinical use. Here human neuronal models may be the most promising avenue regarding the development of novel therapeutic interventions.

\section{REFERENCES}

Ardehali, M. B., Mei, A., Zobeck, K. L., Caron, M., Lis, J. T., and Kusch, T. (2011). Drosophila Set1 is the major histone $\mathrm{H} 3$ lysine 4 trimethyltransferase with role in transcription. EMBO J. 30, 2817-2828. doi: 10.1038/emboj.2011.194

Berger, S. L. (2007). The complex language of chromatin regulation during transcription. Nature 447, 407-412. doi: 10.1038/nature05915

Birnbaum, R., and Weinberger, D. R. (2017). Genetic insights into the neurodevelopmental origins of schizophrenia. Nat. Rev. Neurosci. 18, 727-740. doi: 10.1038/nrn.2017.125

Bledau, A. S., Schmidt, K., Neumann, K., Hill, U., Ciotta, G., Gupta, A., et al. (2014). The H3K4 methyltransferase Setd1a is first required at the epiblast stage, whereas Setd1b becomes essential after gastrulation. Development 141, 1022-1035. doi: 10.1242/dev.098152

Brown, D. A., Di Cerbo, V., Feldmann, A., Ahn, J., Ito, S., Blackledge, N. P., et al. (2017). The SET1 complex selects actively transcribed target genes via multivalent interaction with CpG island chromatin. Cell Rep. 20, 2313-2327. doi: 10.1016/j.celrep.2017.08.030

Cameron, D., Blake, D. J., Bray, N. J., and Hill, M. J. (2019). Transcriptional changes following cellular knockdown of the schizophrenia risk gene SETD1A are enriched for common variant association with the disorder. Mol. Neuropsychiatry 5, 109-114. doi: 10.1159/000497181

Clouaire, T., Webb, S., Skene, P., Illingworth, R., Kerr, A., Andrews, R., et al. (2012). Cfp1 integrates both CpG content and gene activity for accurate H3K4me3 deposition in embryonic stem cells. Genes Dev. 26, 1714-1728. doi: $10.1101 / \mathrm{gad} .194209 .112$

Crump, N. T., and Milne, T. A. (2019). Why are so many MLL lysine methyltransferases required for normal mammalian development? Cell. Mol. Life Sci. 76, 2885-2898. doi: 10.1007/s00018-019-03143-z

Elgersma, Y., and Silva, A. J. (1999). Molecular mechanisms of synaptic plasticity and memory. Curr. Opin. Neurobiol. 9, 209-213. doi: 10.1016/s09594388(99)80029-4

Ernst, C. (2016). Proliferation and differentiation deficits are a major convergence point for neurodevelopmental disorders. Trends Neurosci. 39, 290-299. doi: $10.1016 /$ j.tins.2016.03.001

Ernst, P., and Vakoc, C. R. (2012). WRAD: enabler of the SET1-family of H3K4 methyltransferases. Brief Funct. Genomics 11, 217-226. doi: 10.1093/bfgp/els017

Frega, M., Selten, M., Mossink, B., Keller, J. M., Linda, K., Moerschen, R., et al. (2020). Distinct pathogenic genes causing intellectual disability and autism exhibit a common neuronal network hyperactivity phenotype. Cell Rep. 30, 173-186.e176. doi: 10.1016/j.celrep.2019.12.002

Gabriele, M., Lopez Tobon, A., D'agostino, G., and Testa, G. (2018). The chromatin basis of neurodevelopmental disorders: rethinking dysfunction along the molecular and temporal axes. Prog. Neuropsychopharmacol. Biol. Psychiatry 84, 306-327. doi: 10.1016/j.pnpbp.2017.12.013

Geschwind, D. H., and Levitt, P. (2007). Autism spectrum disorders: developmental disconnection syndromes. Curr. Opin. Neurobiol. 17, 103-111. doi: 10.1016/j.conb.2007.01.009

Hamm, J. P., Shymkiv, Y., Mukai, J., Gogos, J. A., and Yuste, R. (2020). Aberrant cortical ensembles and schizophrenia-like sensory phenotypes in

\section{AUTHOR CONTRIBUTIONS}

SW, AB, TK, NN, J-RVR, and DS designed and wrote the review. All authors contributed to the article and approved the submitted version.

\section{FUNDING}

This work was supported by ERA-NET NEURON-102 SYNSCHIZ-NWO (Nederlandse Organisatie voor Wetenschappelijk Onderzoek) and Hersenstichting (grant number 013-17-003 4538) to DS.

setdla $^{+/-}$mice. Biol. Psychiatry 88, 215-223. doi: 10.1016/j.biopsych.2020 01.004

Jaaskelainen, I. P., Ahveninen, J., Andermann, M. L., Belliveau, J. W., Raij, T., and Sams, M. (2011). Short-term plasticity as a neural mechanism supporting memory and attentional functions. Brain Res. 1422, 66-81. doi: 10.1016/j. brainres.2011.09.031

Kim, J., Kim, J. A., Mcginty, R. K., Nguyen, U. T., Muir, T. W., Allis, C. D., et al. (2013). The n-SET domain of Set1 regulates H2B ubiquitylation-dependent H3K4 methylation. Mol. Cell 49, 1121-1133. doi: 10.1016/j.molcel.2013. 01.034

Kleefstra, T., Kramer, J. M., Neveling, K., Willemsen, M. H., Koemans, T. S., Vissers, L. E., et al. (2012). Disruption of an EHMT1-associated chromatinmodification module causes intellectual disability. Am. J. Hum. Genet. 91, 73-82. doi: 10.1016/j.ajhg.2012.05.003

Kummeling, J., Stremmelaar, D. E., Raun, N., Reijnders, M. R. F, Willemsen, M. H., Ruiterkamp-Versteeg, M., et al. (2020). Characterization of SETD1A haploinsufficiency in humans and Drosophila defines a novel neurodevelopmental syndrome. Mol. Psychiatry 26, 2013-2024. doi: 10.1038/s41380-020-0725-5

Kusch, T. (2012). Histone H3 lysine 4 methylation revisited. Transcription 3, 310-314. doi: 10.4161/trns.21911

Lee, J. H., and Skalnik, D. G. (2008). Wdr82 is a C-terminal domain-binding protein that recruits the Setd1A Histone H3-Lys4 methyltransferase complex to transcription start sites of transcribed human genes. Mol. Cell. Biol. 28, 609-618. doi: 10.1128/MCB.01356-07

Li, F., Huarte, M., Zaratiegui, M., Vaughn, M. W., Shi, Y., Martienssen, R., et al. (2008). Lid2 is required for coordinating $\mathrm{H} 3 \mathrm{~K} 4$ and $\mathrm{H} 3 \mathrm{~K} 9$ methylation of heterochromatin and euchromatin. Cell 135, 272-283. doi: 10.1016/j.cell.2008. 08.036

Li, Y., and Jiao, J. (2017). Histone chaperone HIRA regulates neural progenitor cell proliferation and neurogenesis via beta-catenin. J. Cell Biol. 216, 1975-1992. doi: $10.1083 /$ jcb. 201610014

Lo, L. H., and Lai, K. O. (2020). Dysregulation of protein synthesis and dendritic spine morphogenesis in ASD: studies in human pluripotent stem cells. Mol. Autism 11:40. doi: 10.1186/s13229-020-00349-y

Matsumura, Y., Nakaki, R., Inagaki, T., Yoshida, A., Kano, Y., Kimura, H., et al. (2015). H3K4/H3K9me3 bivalent chromatin domains targeted by lineagespecific DNA methylation pauses adipocyte differentiation. Mol. Cell 60, 584-596. doi: 10.1016/j.molcel.2015.10.025

Min Ko, J., Cho, J. S., Yoo, Y., Seo, J., Choi, M., Chae, J. H., et al. (2017) Wiedemann-steiner syndrome with 2 novel KMT2A mutations. J. Child Neurol. 32, 237-242. doi: 10.1177/0883073816674095

Mohan, M., Herz, H. M., Smith, E. R., Zhang, Y., Jackson, J., Washburn, M. P., et al. (2011). The COMPASS family of H3K4 methylases in Drosophila. Mol. Cell. Biol. 31, 4310-4318. doi: 10.1128/MCB.06092-11

Mossink, B., Negwer, M., Schubert, D., and Nadif Kasri, N. (2021). The emerging role of chromatin remodelers in neurodevelopmental disorders: a developmental perspective. Cell. Mol. Life Sci. 78, 2517-2563. doi: 10.1007/s00018-020-03714-5

Mukai, J., Cannavo, E., Crabtree, G. W., Sun, Z., Diamantopoulou, A., Thakur, P., et al. (2019). Recapitulation and reversal of schizophrenia-related phenotypes 
in setdla-deficient mice. Neuron 104, 471-487.e12. doi: 10.1016/j.neuron.2019. 09.014

Nagahama, K., Sakoori, K., Watanabe, T., Kishi, Y., Kawaji, K., Koebis, M., et al. (2020). Setdla insufficiency in mice attenuates excitatory synaptic function and recapitulates schizophrenia-related behavioral abnormalities. Cell Rep. 32:108126. doi: 10.1016/j.celrep.2020.108126

Nishiyama, J. (2019). Plasticity of dendritic spines: molecular function and dysfunction in neurodevelopmental disorders. Psychiatry Clin. Neurosci. 73, 541-550. doi: 10.1111/pcn.12899

Owen, M. J., Sawa, A., and Mortensen, P. B. (2016). Schizophrenia. Lancet 388, 86-97. doi: 10.1016/S0140-6736(15)01121-6

Ruthenburg, A. J., Allis, C. D., and Wysocka, J. (2007). Methylation of lysine 4 on histone H3: intricacy of writing and reading a single epigenetic mark. Mol. Cell 25, 15-30. doi: 10.1016/j.molcel.2006.12.014

Satterstrom, F. K., Kosmicki, J. A., Wang, J., Breen, M. S., De Rubeis, S., An, J. Y., et al. (2020). Large-scale exome sequencing study implicates both developmental and functional changes in the neurobiology of autism. Cell 180, 568-584.e23. doi: 10.1016/j.cell.2019.12.036

Semple, B. D., Blomgren, K., Gimlin, K., Ferriero, D. M., and NobleHaeusslein, L. J. (2013). Brain development in rodents and humans: identifying benchmarks of maturation and vulnerability to injury across species. Prog. Neurobiol. 106-107, 1-16. doi: 10.1016/j.pneurobio.2013.04.001

Shi, Y., Inoue, H., Wu, J. C., and Yamanaka, S. (2017). Induced pluripotent stem cell technology: a decade of progress. Nat. Rev. Drug Discov. 16, 115-130. doi: $10.1038 / \mathrm{nrd} .2016 .245$

Singh, T., Kurki, M. I., Curtis, D., Purcell, S. M., Crooks, L., Mcrae, J., et al. (2016). Rare loss-of-function variants in SETD1A are associated with schizophrenia and developmental disorders. Nat. Neurosci. 19, 571-577. doi: 10.1038/nn.4267

Sobreira, N., Brucato, M., Zhang, L., Ladd-Acosta, C., Ongaco, C., Romm, J., et al. (2017). Patients with a Kabuki syndrome phenotype demonstrate DNA methylation abnormalities. Eur. J. Hum. Genet. 25, 1335-1344. doi: 10.1038/s41431-017-0023-0

Sohal, V. S., and Rubenstein, J. L. R. (2019). Excitation-inhibition balance as a framework for investigating mechanisms in neuropsychiatric disorders. Mol. Psychiatry 24, 1248-1257. doi: 10.1038/s41380-019-0426-0

Sun, Y., Hu, G., Liu, H., Zhang, X., Huang, Z., Yan, H., et al. (2017). Further delineation of the phenotype of truncating KMT2A mutations: the extended Wiedemann-Steiner syndrome. Am. J. Med. Genet. A 173, 510-514. doi: 10.1002/ajmg.a.38025

Takata, A., Xu, B., Ionita-Laza, I., Roos, J. L., Gogos, J. A., and Karayiorgou, M. (2014). Loss-of-function variants in schizophrenia risk and SETD1A as a candidate susceptibility gene. Neuron 82, 773-780. doi: 10.1016/j.neuron.2014. 04.043

Tyagi, S., Chabes, A. L., Wysocka, J., and Herr, W. (2007). E2F activation of S phase promoters via association with HCF-1 and the MLL family of histone H3K4 methyltransferases. Mol. Cell 27, 107-119. doi: 10.1016/j.molcel.2007. 05.030

Vallianatos, C. N., and Iwase, S. (2015). Disrupted intricacy of histone H3K4 methylation in neurodevelopmental disorders. Epigenomics 7, 503-519. doi: 10.2217/epi.15.1

Yu, X., Yang, L., Li, J., Li, W., Li, D., Wang, R., et al. (2019). De novo and inherited SETD1A variants in early-onset epilepsy. Neurosci. Bull. 35, 1045-1057. doi: 10.1007/s12264-019-00400-w

Zhang, L., Yang, X., Yang, S., and Zhang, J. (2011). The Wnt /beta-catenin signaling pathway in the adult neurogenesis. Eur. J. Neurosci. 33, 1-8. doi: $10.1111 /$ j.1460-9568.2010.7483.x

Conflict of Interest: The authors declare that the research was conducted in the absence of any commercial or financial relationships that could be construed as a potential conflict of interest.

Publisher's Note: All claims expressed in this article are solely those of the authors and do not necessarily represent those of their affiliated organizations, or those of the publisher, the editors and the reviewers. Any product that may be evaluated in this article, or claim that may be made by its manufacturer, is not guaranteed or endorsed by the publisher.

Copyright (C) 2021 Wang, Bleeck, Nadif Kasri, Kleefstra, van Rhijn and Schubert. This is an open-access article distributed under the terms of the Creative Commons Attribution License (CC BY). The use, distribution or reproduction in other forums is permitted, provided the original author(s) and the copyright owner(s) are credited and that the original publication in this journal is cited, in accordance with accepted academic practice. No use, distribution or reproduction is permitted which does not comply with these terms. 